\title{
Influence of vertex region on spin dynamics in artificial Kagome spin ice
}

\author{
Wonbae Bang,,${ }^{1,2, *}$ James Sturm,${ }^{3}$ Raffaele Silvani, ${ }^{4}$ Mojtaba T. Kaffash, ${ }^{3}$ Axel \\ Hoffmann, ${ }^{2, \dagger}$ John B. Ketterson, ${ }^{1}$ Federico Montoncello, ${ }^{5}, \ddagger$ and M. Benjamin Jungfleisch ${ }^{3,}$, \\ ${ }^{1}$ Department of Physics and Astronomy, Northwestern University, Evanston, Illinois 60208, USA \\ ${ }^{2}$ Materials Science Division, Argonne National Laboratory, Argonne, Illinois 60439, USA \\ ${ }^{3}$ Department of Physics and Astronomy, University of Delaware, Newark, DE 19716, USA \\ ${ }^{4}$ Dipartimento di Fisica e Geologia, Università di Perugia, Perugia, Italy \\ ${ }^{5}$ Dipartimento di Fisica e Scienze della Terra, Università di Ferrara, Ferrara, Italy
}

(Dated: June 1, 2020)

\begin{abstract}
We present experimental and theoretical studies of spin-wave mode dynamics in artificial Kagome spin ice vertices made of three identical $15-\mathrm{nm}$ thick elongated $\mathrm{Ni}_{80} \mathrm{Fe}_{20}$ nano-islands (macrospins). We consider several possible configurations, from completely disjointed macrospins (full dipolar inter-element interactions) to fully jointed macrospins (full dipolar-exchange interactions). Using angular-resolved magnetic field dependent broadband ferromagnetic resonance (FMR), we demonstrate the occurrence of a mode localized in the vertex region as indicated by the distinct behavior of the FMR spectra at different angles and configurations. Theoretical calculations using micromagnetic simulations support the existence, origin, and behavior of this mode by interpreting it as a localized, quasi-uniform Kittel mode. Our findings pave the way for designing the most appropriate network consisting of ferromagnetic nanomagnets for specific application purposes in magnonics.
\end{abstract}

\section{INTRODUCTION}

Artificial spin ice (ASI) networks were introduced as auxiliary model systems to simulate elementary atomic spin arrangements at a larger scale. They have become an independent research subject in the field of magnonics - a research area that studies spin waves in magnetic micro- and nanostructures - since they consist of periodic arrangements of magnetic nanoelements, i.e., they are the ideal framework for the study of spin wave Bragg diffraction ${ }^{1-9}$.

These magnetic nanoelements are given an elongated shape such as to facilitate a bi-stable magnetic behavior, in analogy to the spin-up/spin-down behavior of atomic spins. For this reason they are referred to as macrospins ${ }^{10}$. The macrospins mainly interact close to the vertex region by dipolar interaction and, when in contact, also by exchange interaction ${ }^{11-15}$. The detailed understanding of either their static magnetic configurations, mimicking the frustrated state of a crystalline spin ice, and particularly their dynamic behavior, with the ability to control spin wave propagation channels, is currently attracting increasing attention: In recent years, numerous works on ASI have focused on different aspects of either the statics (magnetic configurations) ) $^{5,16,17}$ or the dynamics (spin modes) ${ }^{18-20}$ in the networks. In these most recent works, ASI have been realized by disjointed macrospins or jointed macrospins. However, the main focus of these previous works on jointed networks were issues related to macrospin switching, and studies on the dynamics the main findings were concerned with magnetization oscillations at the center of each macrospin. Presumably, this can be attributed to the fact that the largest signal in the experiments originates from this 'bulk'-like mode, while the distinct dynamics at the vertex when two or more lattice elements are put into contact have mostly been overlooked so far. In terms of contacted macrospins, Bhat et al. discussed the mode dynamics and the importance of the links among the macrospins, but no evidence (theoretical or experimental) for the existence of specific oscillations localized at the vertices were found ${ }^{11}$. Other works on jointed macrospin lattices mainly focused on the formation of

(a)

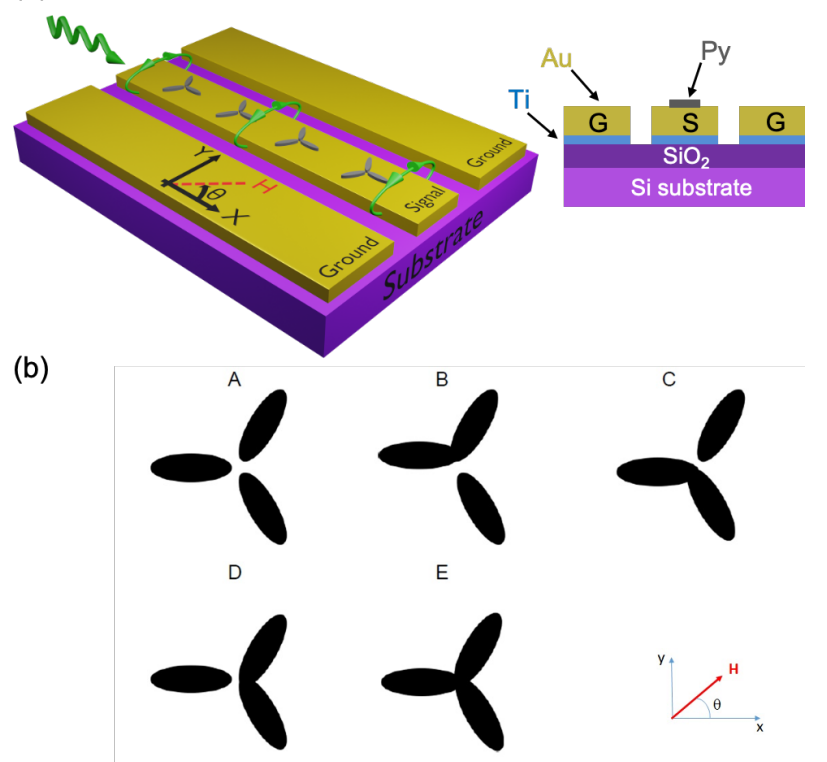

FIG. 1. (a) Sketch of experimental setup. Angular-resolved magnetic field dependence of ferromagnetic resonance is recorded using a coplanar waveguide. The vertex macrospins are patterned directly on top of the signal line. The right panel shows the different thin-film layers as described in the text. Note that the thicknesses are not to scale. (b) Different configurations for the vertex macrospins explored in experiment and micromagnetics. 
domain walls leading to macrospin reversal across the network and the involved statics, and less so on the spinwave dynamics. ${ }^{12-14}$ Furthermore, Montoncello et al. discussed the connection between the domain wall charge drift and a localized soft spin-wave mode in the context of magnetization reversal. ${ }^{15}$

Here, we show the existence of a specific mode relevant to the contact area between two or more macrospins. This mode is localized at the vertex center and contributes to a distinct behavior in the ferromagnetic resonance (FMR) spectra, which allows us to deduce the macrospin configuration just by inspecting the FMR spectra. It is found that the existence of this peculiar mode is critically dependent on the in-plane field orientation. We describe its behavior depending on the geometric features chosen either at the design stage (connecting ASI elements in the fabrication) or operating stage (variation of the direction of the applied field). Our findings reveal a guideline on how to design the most appropriate network consisting of ferromagnetic nanomagnets for specific magnonic device applications such as spin-wave conduits or microwave filters.

\section{SAMPLE GEOMETRY AND MICROMAGNETIC SIMULATIONS}

To interpret the experimentally acquired spectra, we performed micromagnetic simulations using the graphic processing unit (GPU) accelerated software $\operatorname{mumax}^{3}$ [21]. From a geometrical point of view, the samples consist of three equivalent flat cylinders with a thickness of $15 \mathrm{~nm}$ and an elliptical cross section $\left(500 \times 200 \mathrm{~nm}^{2}\right)$, and were discretized into elemental cells of $5 \times 5 \times 15$ $\mathrm{nm}^{3}$. In the magnonics and spin-ice community, these elements are typically referred to as 'islands'; in the following, we call them macrospins. Standard magnetic parameters for permalloy $\left(\mathrm{Ni}_{80} \mathrm{Fe}_{20}, \mathrm{Py}\right)$ (saturated magnetization $M_{s}=700 \mathrm{kA} / \mathrm{m}$, exchange stiffness parameter $A=10 \mathrm{pJ} / \mathrm{m}$, gyromagnetic ratio $\gamma=$ $185 \mathrm{rad} \mathrm{GHz} / \mathrm{T})$ were used. We studied five different configuration/arrangements of macrospins as shown in Fig. 1: in the first configuration the elements are completely separated (Type A). Three configurations (Type B, C, D) have two out of three ellipses overlapping at the inner edges, and in the last configuration all three ellipses are overlapping at the inner edges (Type E), i.e., the elements are connected at the vertex.

To break any artificial symmetry due to the micromagnetic approach and meet the realistic experimental conditions, we considered a constant angular offset of $+1^{\circ}$ (hence, corresponding to the nominal angle of $0^{\circ}$ ). The calculations considered a magnetic field $\mu_{0} H$ going from $-150 \mathrm{mT}$ to $150 \mathrm{mT}$ in steps of $\Delta H=1 \mathrm{mT}$. For each field value, a uniform magnetic field pulse:

$$
b(t)=b \frac{\sin 2 \pi f_{0}\left(t-t_{0}\right)}{2 \pi f_{0}\left(t-t_{0}\right)}
$$

with $b=10 \mathrm{mT}$ and $f_{0}=15 \mathrm{GHz}$ was applied in the outof-plane direction to excite the eigenmodes in the ASI system. In a subsequent step, the dispersion relation was obtained by the absolute value of the Fast Fourier Transform for the time evolution of the out-of-plane average magnetization. The spatial profiles that we will show in the following are the real part of the Fast Fourier Transform of each cell in the simulated area at a given combination of frequency and externally applied field.

\section{EXPERIMENTAL METHODS}

In the following, the sample fabrication and ferromagnetic resonance (FMR) measurement technique are introduced.

\section{Sample fabrication}

The samples were fabricated in the following fashion. In the first step, a 50- $\Omega$ matched coplanar waveguide (CPW) made of $\mathrm{Ti} / \mathrm{Au}(5 \mathrm{~nm} / 120 \mathrm{~nm})$ was fabricated by using electron beam evaporation and photolithography on intrinsic $\mathrm{Si}$ substrates having a $300 \mathrm{~nm} \mathrm{SiO}_{2}$ layer; the latter insures electrical isolation from the CPW; see Fig. 1. The signal line at the center of the CPWs has a width of $20 \mu \mathrm{m}$, and the two ground lines are $40-\mu \mathrm{m}$ wide, while the spacing between the central line and each ground line is $8 \mu \mathrm{m}$. Arrays of artificial Kagome spin ice vertices (a distance of $1.39 \mu \mathrm{m}$ between individual vertices was chosen so that they do not interact) were defined on the top of the signal line using electron beam lithography ${ }^{22}$. A double layer positive resist of polymethyl methacrylate was spin-coated on the CPW prior to electron beam exposure. After exposure and development, thin $\mathrm{Ti}$ and $\mathrm{Au}$ adhesion layers were deposited together with 15-nm of $\mathrm{Py}$ without capping layer using electron beam evaporation followed by lift-off, thereby creating three identical elongated islands to form artificial Kagome spin-ice vertices. A sketch of the thin-film layer stack is shown as an inset in Fig. 1(a). The thicknesses of the deposited materials were monitored by a quartz crystal microbalance during the evaporation: the rates were $0.2 \AA / \mathrm{sec}$ for $\mathrm{Ti}, 1.4 \AA / \mathrm{sec}$ for $\mathrm{Au}$, and 0.4 $\AA /$ sec for Py, respectively. The pressure in the chamber during the metal depositions was $3 \times 10^{-7}$ Torr.

\section{Details on FMR measurements}

The experimental data were obtained using a fully automated home-made microwave probe station that allows for a variation of the in-plane magnetic field. A vector network analyzer (Keysight N5225A) connected via picoprobes to the CPW (Fig. 1) was used to record the transmission parameter S21. A background signal at a large magnetic field (above the measured field range) 

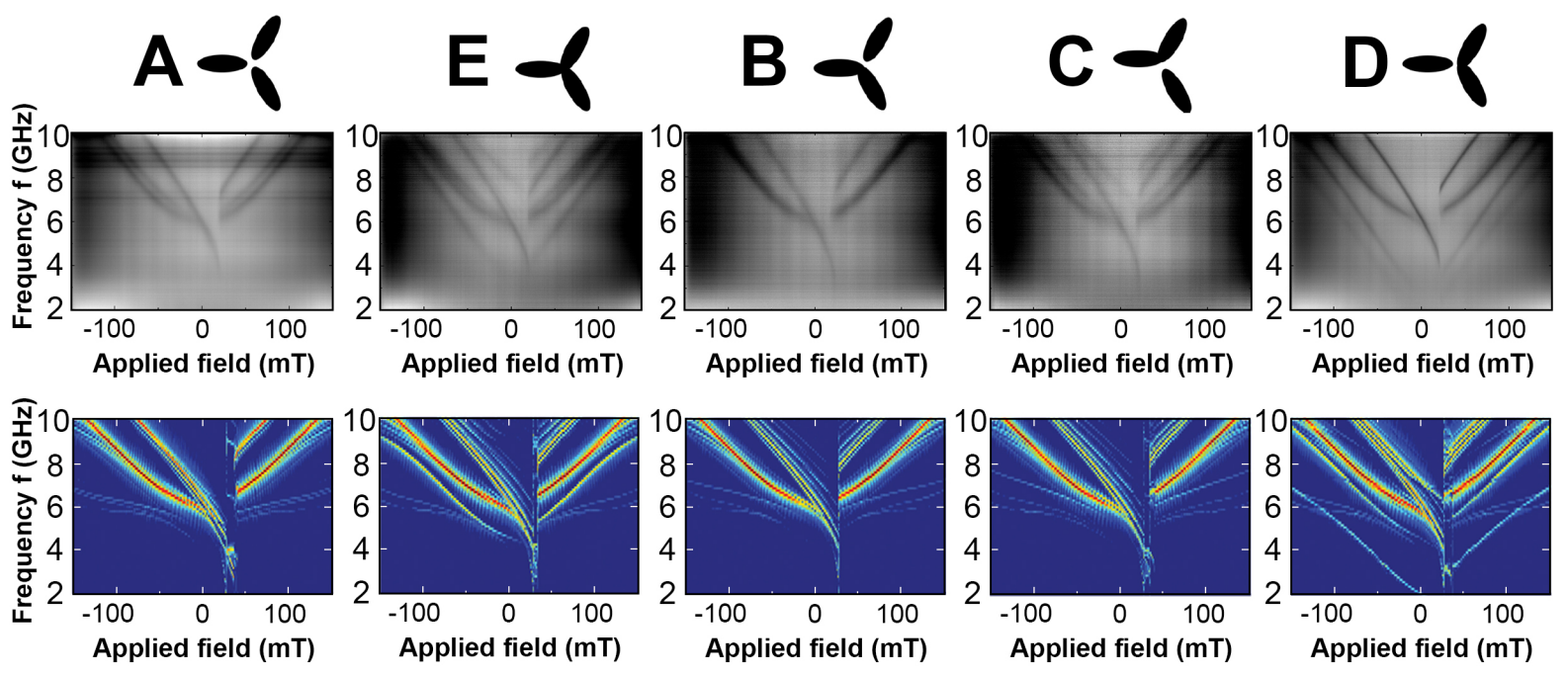

FIG. 2. Top illustrations: (A) - (D) various artificial Kagome spin-ice vertices studied by broadband ferromagnetic resonance (FMR) and micromagnetic simulations. Middle panel: False color-coded images of the experimentally acquired FMR spectra measured at $\theta=0^{\circ}$, see field direction $\mu_{0} H$ with respect to the vertex in inset above top panel. In $\theta=0^{\circ}$ configuration, due to symmetry, the vertex center mode (VCM) appears only in case E and D. Lower panel shows the results of the corresponding micromagnetic simulations in color-coded images.

was taken and subtracted from the actual spectra. The magnetic-field independent signal, visible as horizontal lines at higher frequencies are due to the subtraction of this background spectrum. However, we point out that these field-independent traces do not affect the analysis and interpretation of the experimental data.

\section{RESULTS}

The experimental FMR results at $0^{\circ}$ are shown in Fig. 2 (middle panel). In general, the main curves in the spectra are due to the fundamental $(\mathrm{F})$ modes of each macrospin ${ }^{23,24}$, and depending on the orientation of the macrospin with respect to the applied magnetic field direction and the consequent demagnetizing fields, the lowest frequency curve belongs to the macrospin that is the least aligned with the field direction [i.e. the elliptical island with the largest angle of its major axes with respect to the applied magnetic field, as can be seen in Fig. 3(a) and (c)], while the highest frequency mode corresponds to the most aligned one [Fig. 3 (b) and (d)].

In Ref. [24] we introduced the effective gyromagnetic ratio as

$$
\gamma_{e f f}=\frac{\partial \omega}{\partial B}=\frac{2 \pi}{\mu_{0}} \frac{\partial f}{\partial H},
$$

which offers a practical way to analyzing the FMR spectra, where $\omega$ is the angular frequency, $f$ is the linear frequency and $H$ is the magnetic field. At any given $H$, the slope $\frac{\partial f}{\partial H}$ of a FMR curve is an indication of the average direction of the magnetic moment density $<\mathbf{M}>$ inside a macrospin and its evolution as the field is varied. Due to shape anisotropy, a larger slope $\gamma_{\text {eff }}$ corresponds to a more aligned $<\mathbf{M}>$ with respect to the macrospin long axis. Depending on the vertex type (A, B, C, D or E in Fig. 1), at special angles $\left(\theta=0^{\circ}\right.$ and/or $\theta= \pm N \times 60^{\circ}$ with integer $\mathrm{N}$ ) two macrospins occur symmetrically with respect to the given field direction and are hence indistinguishable (at least in principle) from each other: in such cases (e.g., Figs. 2, A and E) there are only two curves detectable in the FMR spectra, one being particularly intense due to the larger magnetic area (two macrospins) involved in the oscillation [i.e., modes in Figs. 3(a) and (c)]. In the others cases, the spectra show three different curves, corresponding to the three fundamental modes localized in each of the three macrospins, being inequivalent. In the FMR spectra of cases B and C (Fig. 2), the splitting of the lowest curve is barely visible, which is consistent with the fact that bulk modes must depend only weakly on the condition of the macrospin edges. Other differences in the measured spectra are due to unavoidable intensity variations caused by a change of the coupling strength of the magnetization and the microwave field generated by the different CPWs, and not due to actual underlying physical effects.

In case $\mathrm{E}$ and $\mathrm{D}$, a new curve appears, which is actually the lowest frequency curve, and will not be interpreted as a bulk mode. Comparison with the micromagnetic 


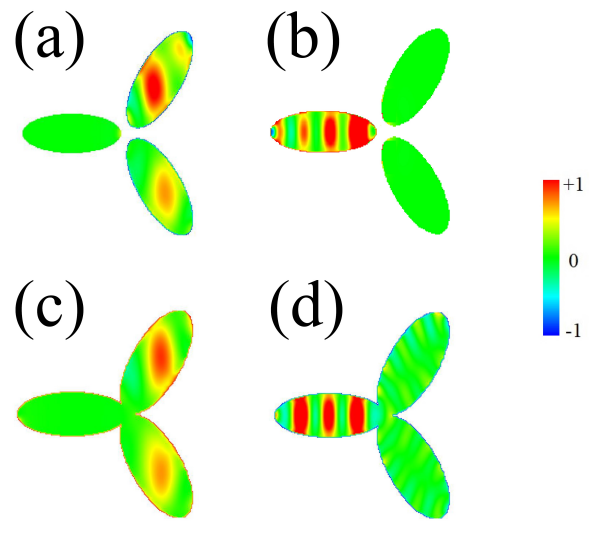

FIG. 3. Type A [insets (a) and (b)] and Type E [insets (c) and (d)] bulk modes for the case $\theta=0^{\circ}$. Being symmetric to the applied field direction, both the oblique macrospins oscillate at the same frequency [insets (a) and (c)], while the macrospin parallel to the field direction oscillates independently, at a higher frequency [panel (b) and (d)]; when no symmetry is present, each macrospin has a different frequency, so that modes (a) or (c) split into two separate ones. The undulation of the mode profile is the consequence of a hybridization, an inherent effect in calculations, particularly evident in insets (b) and (d). All these modes are fundamental in the sense that the undulations never involve negative phases, and hence are expected to give large FMR signals.

simulations (lower panel in Fig. 2) reveals that this curve corresponds to a localized mode residing at the vertex center. The reason why this mode is visible in $\mathrm{D}$ but not in $\mathrm{B}$ or $\mathrm{C}$ will be given below, along with details on the origin and behavior of this mode.

The simulated spectra show more curves aside from the main ones, which must be considered as replica due to higher order modes, i.e., modes having nodal lines, and consequently lower intensity and strength: for this reason, they are not detected in the experiments, though in the simulations they seem to broaden the signal in-
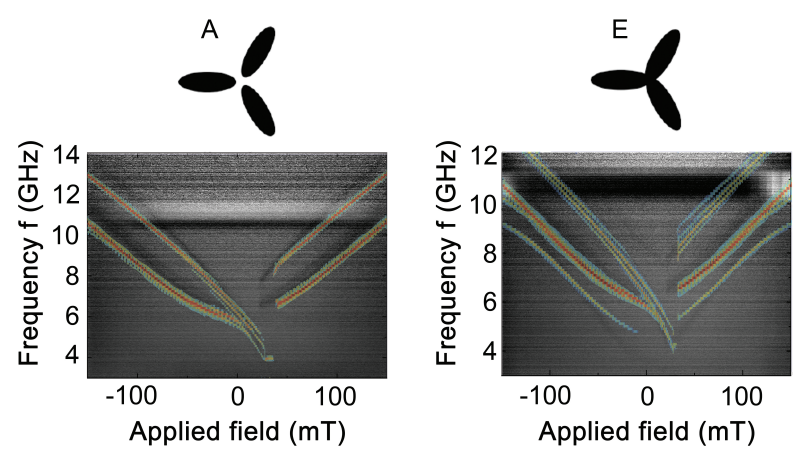

FIG. 4. Comparison between experimental (gray scale) and calculated (lines) $f\left(\mu_{0} H\right)$ curves for separated (Type A, left) and joint (Type E, right) configurations, for nominal $\theta=0^{\circ}$, i.e. applied field parallel to the $x$-direction. tensity [see for example Fig. 2(A), where two curves are expected].

In the simulations, the tiny curves at the lowest frequencies are end modes, localized in narrow regions close to the ends of the macrospins, and that is why they have a weak intensity (barely visible in simulations and not detected at all in the experiments).

To demonstrate the agreement with the experimental FMR data, we plot in Fig. 4 the superposition of the theoretically expected $f\left(\mu_{0} H\right)$ curves (straight lines) and the measured data (false-color coded images) for $\theta=0^{\circ}$ for both systems $\mathrm{A}$ and $\mathrm{E}$. At $H=0$ the three macrospins must be in their Ising-saturated condition (i.e., magnetization aligned with the macrospin axis) and the fundamental modes of the three macrospins should be at the same frequency (at about $6 \mathrm{GHz}$ ). On average this is confirmed by the experimental data. However, as far as the simulations are concerned, at $H=0$ the lower curve [corresponding to the modes (a) or (c) in Fig. 3] is not exactly crossing the upper curve [corresponding to the modes (b) or (d) in Fig. 3]. This can be understood as follows: the micromagnetic sample is not perfectly symmetric for a rotation by $120^{\circ}$, due to the intrinsic incompatibility of this angle with underlying square-cell micromagnetic mesh.

The lowest lying curve in the spectrum of system A shown in Fig. 4(A) (left panel) corresponds to the bulk mode shown in Fig. 3(a), while the upper curve corresponds to Fig. 3(b). On the other hand, the spectrum of system E shown in Fig. 4(E) (right panel) features three dispersion curves, the one in the middle being particularly intense and corresponding to the mode in Fig 3(c), the upper curve corresponding to the mode in Fig 3(d), and the low-lying frequency curve corresponding to a mode localized at the vertex center. This vertex-center mode is discussed in more detail below.

\section{Variation with the applied magnetic field angle}

The spectra were collected with the field at $\theta=$ $0^{\circ}, 15^{\circ}, 30^{\circ}, 45^{\circ}$ (see Figs. 2, 5, 6, 7). Due to symmetry, the cases $B\left(-60^{\circ}\right), C\left(+60^{\circ}\right)$, and $D\left(-180^{\circ}\right)$ would be equivalent and indistinguishable in principle in FMR spectra (apart from experimental errors due to patterning imperfections). In each spectrum, the specific behavior of a frequency curve is an indication of the orientation of the corresponding macrospin (where the oscillation occurs) with respect to the applied magnetic field. As remarked in Refs. [24] and [25], as far as the fundamental modes are concerned, the macrospins behave largely independently of each other, and hence the full FMR spectrum can be thought of as the superposition of the single frequency curves of each single macrospin, as taken alone. In particular, in those works it was demonstrated that, when the field is applied at an angle lower than $45^{\circ}$ with respect to the macrospin axis, the corresponding fundamental mode dispersion is almost linear. 

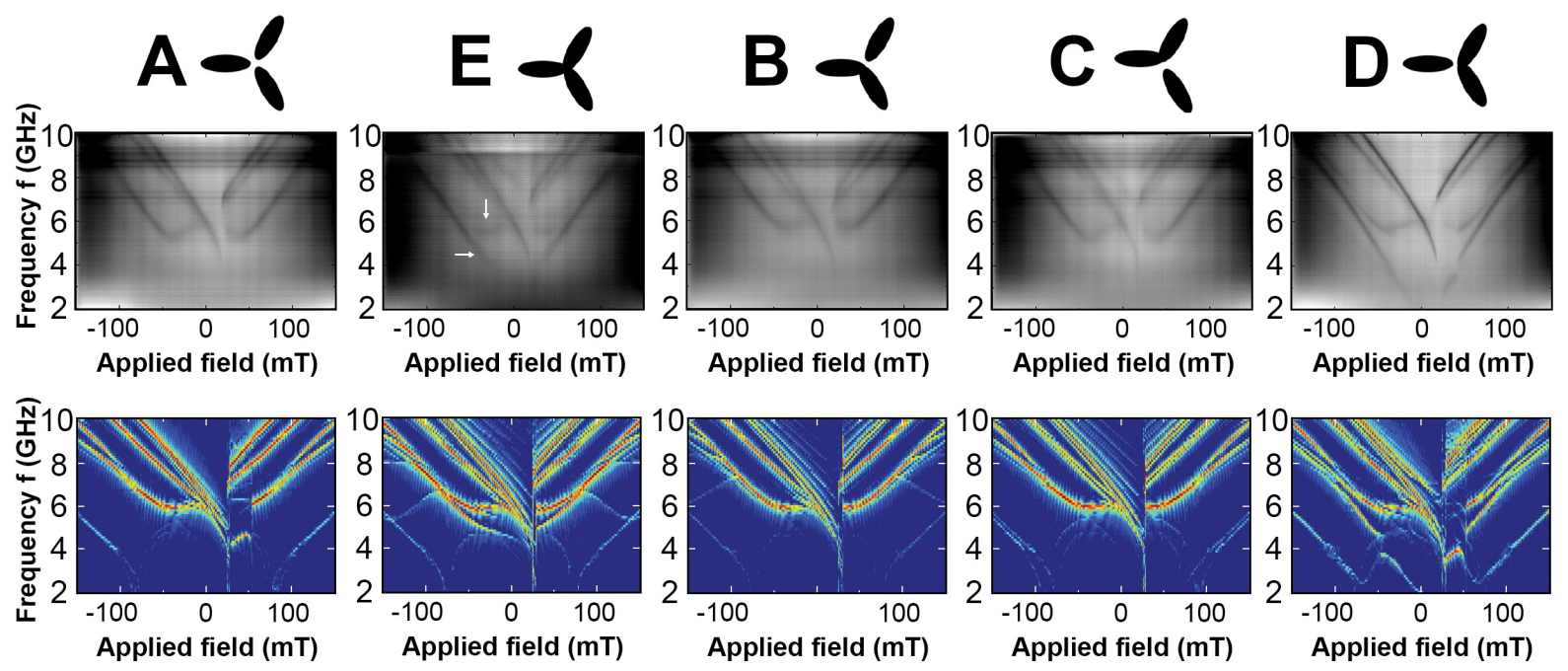

FIG. 5. FMR spectra for nominal $\theta=15^{\circ}$. Top illustrations: (A) - (D) various artificial Kagome spin-ice vertices studied by broadband FMR spectra and micromagnetic simulations. Middle panel: False color-coded images of the experimentally acquired FMR spectra. Lower panel shows the results of the corresponding micromagnetic simulations in color-coded images. White arrows in middle panel $\mathrm{E}$ indicate the experimental evidence for a splitting of the curves discussed in the text and by simulations shown in bottom panel E.

When the angle is around $75^{\circ}$, the curve initially (i.e., at high field values) shows a linear behavior and then (on approaching zero field) reaches a plateau. When the angle gets very close or equal to the critical value of $90^{\circ}$, the frequency curve displays a $W$-shape behavior ${ }^{24,25}$.

If the field is applied at $30^{\circ}$, two of the three macrospins will behave identically, and will be degenerate in frequency, while the other one will behave like a macrospin with the field perpendicular to its axis (hence, producing the typical $W$-shape curve). These observations are of great help in analyzing the FMR spectra qualitatively by eye.

As far as the number of dispersion curves in the FMR spectra are concerned (Figs. 2, 5, 6, and 7), we remark that when the applied field is oriented at $0^{\circ}$, in Type A, $\mathrm{E}$ and $\mathrm{D}$ samples, two out of three macrospins are symmetric and their corresponding fundamental modes degenerate, while the third macrospin is inequivalent and gives its own particular curve, so that one can find two main FMR curves; moreover, if at least two macrospins are in contact (i.e., only for Type E and D) an additional curve is found, relative to the vertex center mode (a localized mode, discussed in detail below). Similarly, at $30^{\circ}$ (Fig. 6) two out of three macrospins are equivalently tilted $30^{\circ}$ with respect to the applied field, while the third one is perpendicular to the field: limiting our discussion to the fundamental modes only, this implies again two main curves. At $15^{\circ}$ (Fig. 5) and $45^{\circ}$ (Fig. 7), the symmetry is broken and we see three curves corresponding to the dispersions of the three independent fundamental modes of the three macrospins, which in these configurations are no longer equivalent. Details of the specific shape of the curves (linear, sub-linear or reaching a minimum before increasing with field again in a "W-shape") are extensively discussed in Ref. [25]. These symmetry considerations are also directly applicable to the experimental data; however, in contrast to the micromagnetic simulations, the effective coupling of the microwave magnetic field changes as the in-plane angle is rotated. This stems from the fact that the direction of the microwave magnetic field is fixed (in $y$-direction), while the direction of $H$ is changed.

\section{The vertex center mode}

The most important finding of this work is the existence of a spin-wave mode localized in the vertex region of sample E (jointed macrospins) that is not present in sample A (entirely separated macrospins), and can be found in sample B, C, D only depending on special conditions discussed below. Our interpretation is that this new localized mode is the Type $\mathrm{E}$ counterpart of the inner end mode [Fig. 8(c)] of sample A, but merged to the vertex center as a consequence of the merging of the macrospins.

What might have been the inner end mode in sample $\mathrm{E}$ has merged at the center into a new, single mode: 


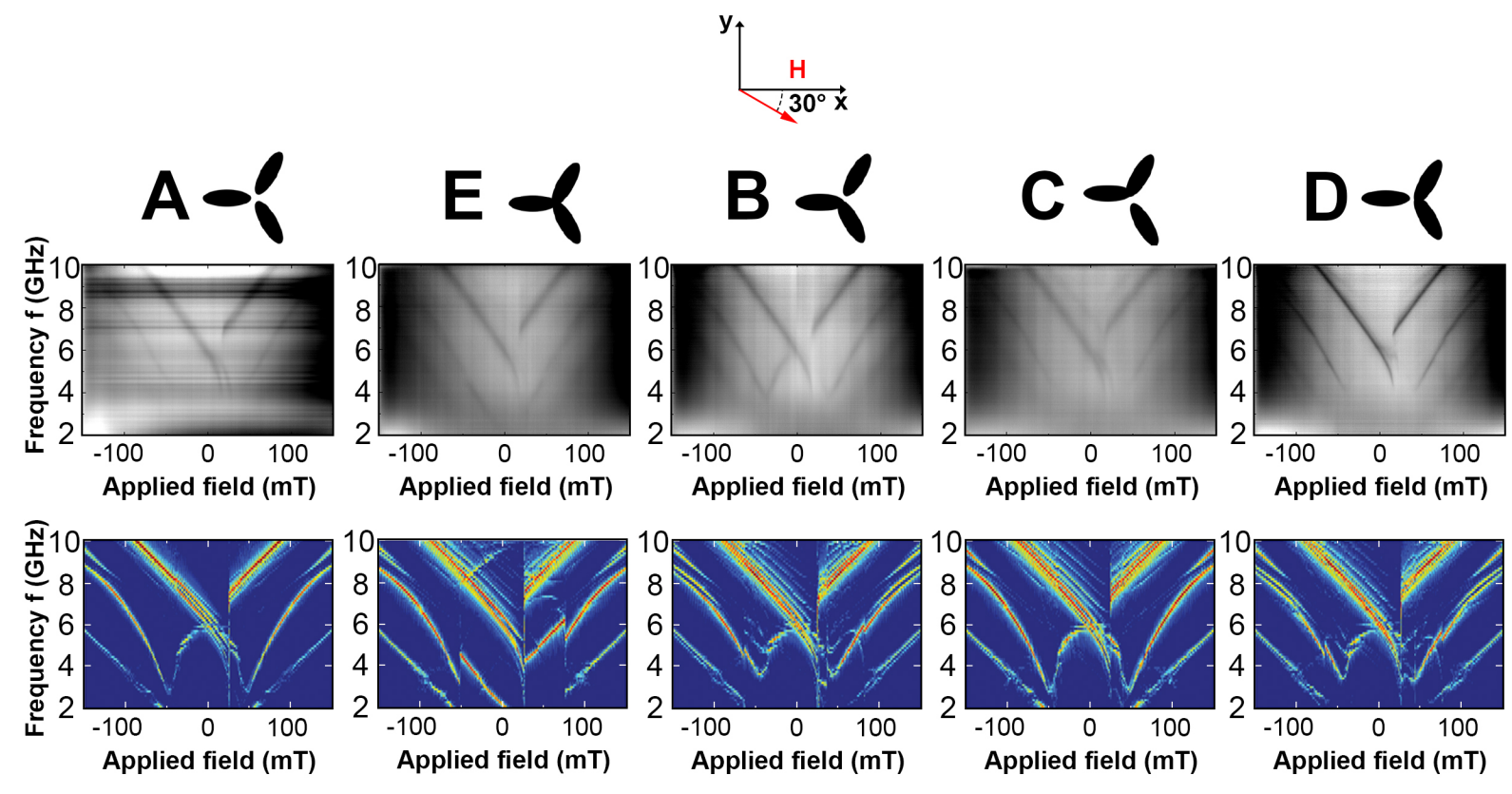

FIG. 6. FMR spectra for nominal $\theta=30^{\circ}$. Top illustrations: (A) - (D) various artificial Kagome spin-ice vertices studied by broadband FMR spectra and micromagnetic simulations. Middle panel: False color-coded images of the experimentally acquired FMR spectra. Lower panel shows the results of the corresponding micromagnetic simulations in color-coded images.
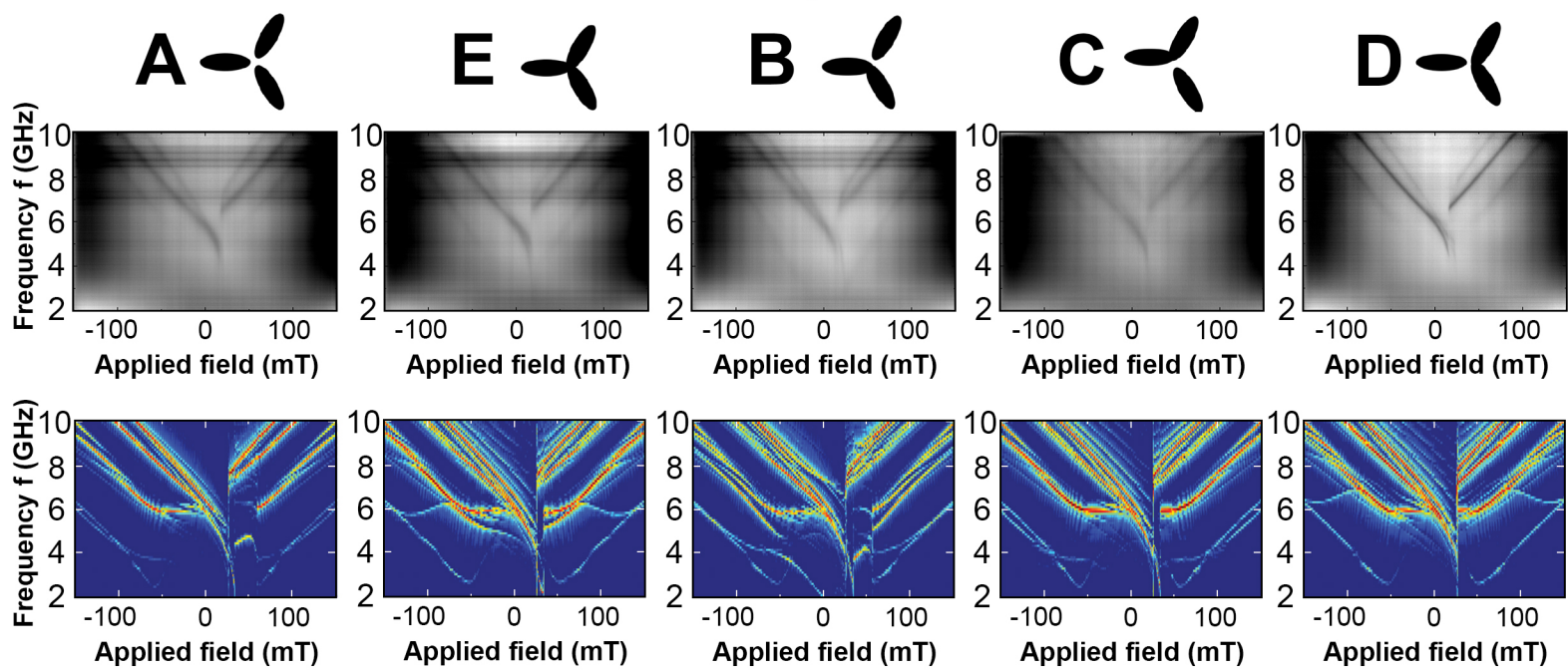

FIG. 7. FMR spectra for nominal $\theta=45^{\circ}$. Top illustrations: (A) - (D) various artificial Kagome spin-ice vertices studied by broadband FMR spectra and micromagnetic simulations. Middle panel: False color-coded images of the experimentally acquired FMR spectra. Lower panel shows the results of the corresponding micromagnetic simulations in color-coded images.

the vertex center mode (VCM). Differently from ordinary end modes localized in narrow regions close to the macrospin ends, the VCM resides in a rather extended region [(Fig. $8(\mathrm{c})]$, and this effect is a consequence of the exchange interaction which prevails over the dipolar one.
The exchange interaction, indeed, increases the coherence of the spin oscillation to a wider area, and allows the occurrence of a quasi-uniform mode localized at the vertex center. This fact also explains the strong signal of VCM in the FMR response, both in the calculations as well 

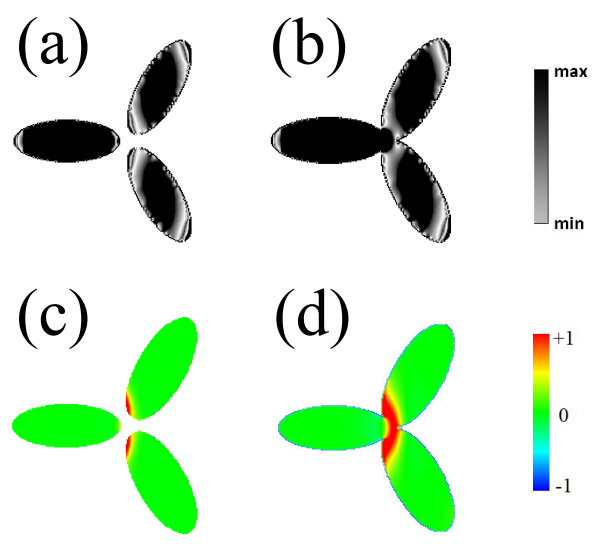

FIG. 8. 2D maps of the internal field profiles calculated at $\mu_{0} H=80 \mathrm{mT}\left(\theta=0^{\circ}\right)$ for (a) Type A and (b) Type E configurations: white corresponds to minimum value, black to maximum value (arb. units); the black color is saturated to allow the visualization of the profile of the minima. Note that these minima correspond to the zone where the magnetization is mostly misaligned to the applied field; in (b) minima at the vertex center are shallower than in (a) and more uniform, due to the action of exchange interaction which keeps the magnetic moments less misaligned, and pushes the mode to a higher frequency with a wider extension. The corresponding spatial profiles of the dynamic magnetization for both cases is shown in (c) and (d).

as in the experiments. Furthermore, the exchange interaction considerably raises the overall VCM frequency in the jointed case $\mathrm{E}$ (around $8 \mathrm{GHz}$ at $\mu_{0} H=-100 \mathrm{mT}$ ), compared to the frequency of the inner end mode in the disjointed case A (around $6 \mathrm{GHz}$ at $\mu_{0} H=-100 \mathrm{mT}$ ). Hence, differently from ordinary end modes of saturated samples ${ }^{26}$, the VCM frequency is very close to the frequency of the bulk modes.

The localization of the low frequency excitations can be explained by the confinement that the magnetic oscillations experience in the minima of the internal effective field $H_{\text {eff }}$ (the sum of the applied field with the demagnetizing and exchange fields). In Fig. 8, we illustrate the strict correlation between $H_{\text {eff }}(x, y)$ [panel (a) and (b)] and the low frequency profile of the dynamic magnetization $\delta m(x, y)$ [Fig. 8, panel (c) and (d)]. Note that in panel (a) the internal field at the inner ends is strongly varying from white to black, and is rather uniform only very close to the ends, consistent with the non-zero amplitude of the mode in panel (c). Conversely, in panel (b) the internal field is more uniform (the grayscale at the inner ends is less varying), which is the reason why the corresponding VCM profile (d) is considerably more extended. This mode was actually detected in the measured spectra of sample E (Fig. 4), while completely absent in sample A. As expected, the frequency at $\mu_{0} H=-100$ $\mathrm{mT}$ is very large (around $8 \mathrm{GHz}$ ) and close to the bulk mode frequencies.

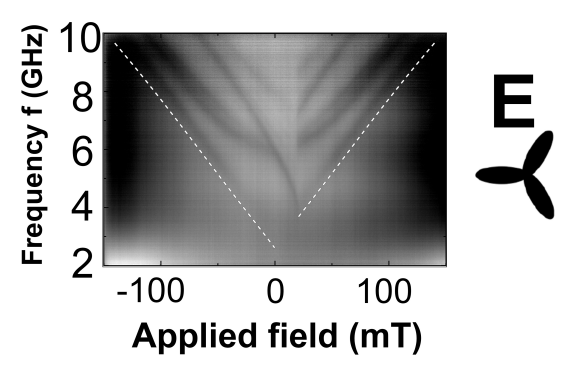

FIG. 9. Theoretical Kittel curve (dashed line) for the mode localized at the vertex center on the experimental (grayscale) FMR dispersion $f\left(\mu_{0} H\right)$ in Type E configurations, for nominal $\theta=0^{\circ}$, i.e. applied field parallel to the $x$-direction. A similar scenario also applies to sample type B, C, and D provided that the existence conditions for this mode are met.

\section{Interpretation of VCM as a localized Kittel mode}

In order to corroborate the localized nature of the VCM, we used the analytical formula for a fundamental Kittel mode ${ }^{27,28}$ with frequency $f=\omega / 2 \pi$ and:

$$
f(H)=\frac{\gamma}{2 \pi} \mu_{0} \sqrt{H_{\text {eff }}(H)\left[H_{\text {eff }}(H)+M_{s}\right]}
$$

using, for each applied field value $H$, the values of the internal effective field $H_{\text {eff }}$ calculated with mumax ${ }^{3}$ in the region where the mode profile has the largest intensity, again as calculated by mumax ${ }^{3}$. We found an almost perfect agreement with the experimental data. The results are plotted in Fig. 9.

\section{VCM existence conditions at different geometric configurations}

As remarked above, the basic existence condition for a VCM is a contact between two macrospins, at least: this
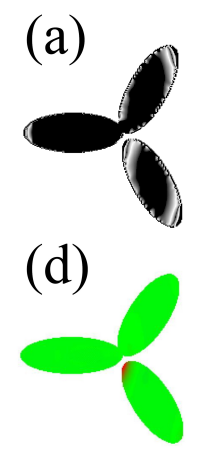

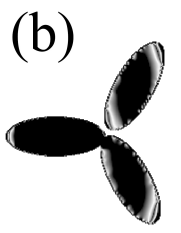

(e)

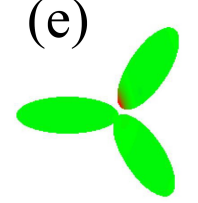

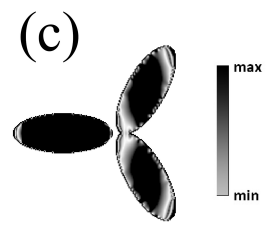

(f)

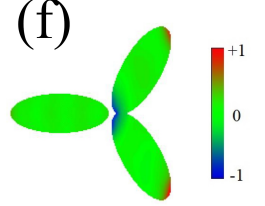

FIG. 10. Internal field space profiles calculated at $\mu_{0} H=$ $-80 \mathrm{mT}\left(\theta=0^{\circ}\right)$ for type B, C and D configurations [(a,d), $(\mathrm{b}, \mathrm{e})$, and $(\mathrm{c}, \mathrm{f})$, respectively]: white corresponds to minimum value, black to maximum value; the black color is saturated to allow the visualization of the profile of the minima. Note the extended white area in type $\mathrm{D}$, minimum of $H_{\text {eff }}(x, y)$. 


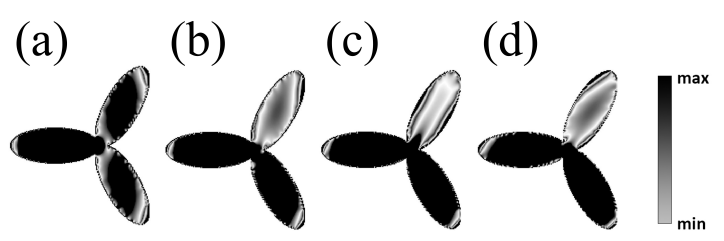

FIG. 11. Internal field $H_{\text {eff }}(x, y)$ space profiles for type E configuration, calculated at $\mu_{0} H=-80 \mathrm{mT}$ directed at (a) $0^{\circ}$, (b) $15^{\circ}$, (c) $30^{\circ}$, (d) $45^{\circ}$; the black color is saturated to allow the visualization of the profile of the minima. Note that $H_{\text {eff }}(x, y)$ has a minimum at the vertex center only in (a), i.e. when $H_{0}$ is applied parallel to a macrospins.

favors an extended area for the minimum of $H_{\text {eff }}(x, y)$ where the mode oscillates with significant amplitude and consequently a strong FMR signal. In Fig. 10 we show the correlation between the vertex configurations of $\mathrm{B}, \mathrm{C}$, and $\mathrm{D}, H_{e f f}(x, y)$ minima [panel (a), (b), (c), respectively] and the localized dynamic modes [end modes in panel (d) and (e), VCM in panel (f)]: crucial to that is the orientation of the disjointed macrospin with respect to the applied field direction $\left(\theta=0^{\circ}\right.$ in the figure).

If type $\mathrm{E}$ and $\mathrm{D}$ configurations are considered (the same arguments can be made for type B and C, provided the field orientation is rotated $\theta= \pm 120^{\circ}$ to realize the type D configuration), the applied field angle becomes even more important for the VCM formation: we investigate the occurrence of $\mathrm{VCM}$ in type $\mathrm{E}$ at the applied field angles $\theta=0,-15^{\circ},-30^{\circ},-45^{\circ},-60^{\circ}$. The VCM forms, and has significant strength, only when the external field is applied parallel to a macrospin in type E configuration [Fig. 11(a)], or to the macrospin that is separated from the others in type D configuration, Fig. 10(c). This can be understood again by considering the internal effective field profiles: in Fig. 11 a minimum effective field localized at the vertex center occurs only in (a), where $H$ is parallel to a macrospin; in Fig. 10 only in type $\mathrm{D}$ configuration, where $H$ is parallel to the separated macrospin. This effect is the largest when the vertex has a mirror symmetry with respect to the field direction. Even in the case when all three macrospins
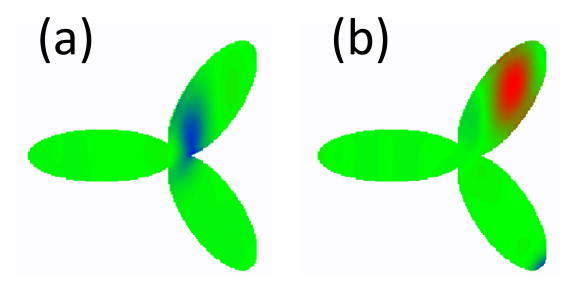

FIG. 12. The splitted modes Mode profiles calculated at $\mu_{0} H=-50 \mathrm{mT}\left(\theta=15^{\circ}\right)$ for type E configuration: frequency of (a) is calculated at $5.1 \mathrm{GHz}$, (b) at $5.6 \mathrm{GHz}$. These modes result from the splitting of the fundamental mode starting above $-70 \mathrm{mT}$. At such angle, the splitting starts above $-70 \mathrm{mT}$. are in contact, the minimum occurs in a region belonging to the ends of the macrospins oblique to the applied field direction (see Fig. 8). Hence, the strength and thus the FMR intensity of type $\mathrm{D}$ and type $\mathrm{E}$ for $\theta=0^{\circ}$ are approximately the same (see Fig. 2).

Note that the fit of the VCM dispersion with the Kittel curve is in excellent agreement for both cases $\mathrm{E}$ and D (also compare to Fig. 9 for case E), and hence it is insensitive to the macrospin parallel to the field that does not contribute to the extension of the internal effective field minima. On the other hand, increasing the in-plane field angle from $\theta=0^{\circ}$ results in progressive distortion of VCM mode and progressive displacements from the vertex center towards the macrospin bulk. Eventually, the VCM mode hybridizes with the bulk mode of the upper macrospin, which gives rise to the lowest frequency curve in the FMR spectra. This means that the VCM mode is no longer an independent mode, but coupled to the bulk mode of the neighboring macrospin. This can also be seen from corresponding variation of the internal field in Fig. 11.

To further support this interpretation, we focus the following discussion on Fig. 5, panel E. Experimentally, we observe that up to around $-70 \mathrm{mT}$ only one, wide FMR curve is present in the spectum, corresponding to a single excitation. Below that field value, as highlighted by the white arrows, the curve splits into two branches. We interpret this effect as the splitting of the upper macrospin fundamental mode into two modes localized within two complementary halves of the same macrospin, i.e. one localized close to the vertex center (corresponding to VCM at a virtual $\theta=0^{\circ}$ ) the other close to the outer end of the macrospin (Fig. 12).

\section{DISCUSSION AND CONCLUSIONS}

We demonstrated how a direct contact among macrospins at a vertex of a Kagome ASI affects the spin dynamics, both by calculations and experiment. The main finding of our work is the observation of a mode localized within the contact area - the vertex center mode (VCM). The VCM arises when at least two macrospins are in contact. This is a necessary but not sufficient condition: in fact, the contact allows the possibility of an internal field well that is large enough and rather uniform (see Fig. 10) to produce an extended localized oscillation (and not pure end modes). Consequently, this leads to a large intensity of the VCM. Another crucial degree of freedom is the field orientation with respect to the vertex: since minima occur in the region where the magnetization is mostly misaligned with the applied field, the VCM strength is the largest when the field is parallel to one of the macrospins (i.e., the ASI vertex has a mirror plane containing the applied field direction). The macrospin parallel to the field plays a minor role in the VCM localization, meaning that it is not important whether it is in contact at the vertex or not. On the other hand, it is 


\begin{tabular}{c|c|c}
\hline \hline & $\Delta f_{\Gamma M}$ & $v_{g}$ \\
$\mu_{0} H=0.08 \mathrm{~T}$ & $8.0 \mathrm{MHz}$ & $6.2 \mathrm{~m} / \mathrm{s}$ \\
$\mu_{0} H=0.1 \mathrm{~T}$ & $5.0 \mathrm{MHz}$ & $3.9 \mathrm{~m} / \mathrm{s}$ \\
\hline \hline
\end{tabular}

TABLE I. Frequency bandwidth $\Delta f_{\Gamma M}$ and average spin-wave group velocity $v_{g}$ of a propagating $\mathrm{VCM}$ in a periodic ASI at different field values.

important that the other two macrospins (oblique with respect to the field) are in contact with each other.

When considering dense arrays (with a larger number of macrospins per fixed volume), we note that as the nanoelement size is lowered, the internal field becomes so inhomogeneous that the formation of a fundamental-like mode is not possible anymore. This is because of a lack of a plateau in the internal field distribution, and as a result the mode is replaced by end modes which acquire extension and intensity due to the increased spin oscillation coherence favored by the exchange interaction ${ }^{29,30}$. Keeping the same micromagnetic elemental cell for direct comparison, we tested this scenario and calculated the modes of the Type E-like system at 0 degrees. We did not find a "fundamental-like" mode when the lateral size of the original macrospins is reduced to $20 \%$ $\left(100 \times 40 \mathrm{~nm}^{2}\right.$, i.e., a fifth of its original size), but we do find end modes contributing with a large signal. The inner end mode corresponds to the VCM, which is more extended and hence it dominates even more the low frequency dynamics than at a larger lateral macrospin size.

Our findings are particularly important when designing a ASI geometry with the purpose of tailoring the specific dynamic features (such as microwave filters or magnonic crystals): in a fully periodic ASI, a collective mode of the system is always of the Bloch form:

$$
\delta \mathbf{m}_{\mathbf{k}}(\mathbf{r})=\delta \widetilde{\mathbf{m}}_{\mathbf{k}}(\mathbf{r}) e^{i \mathbf{k} \cdot \mathbf{r}},
$$

where $\mathbf{r}$ is the position vector and $\mathbf{k}$ the wavevector. Any of the modes described for the isolated vertex can be the cell function $\delta \widetilde{\mathbf{m}}_{\mathbf{k}}(\mathbf{r})$ of a Bloch wave in the vertex array, and, depending on how strong the dynamic stray fields created by the mode oscillation are, the dispersion bandwidth can be small or large. Even a localized mode can show an appreciable bandwidth, provided a significant amplitude and considerable spatial extension ${ }^{31,32}$. As we have demonstrated above, the VCM happens to fulfill all of those conditions.

This means we can find a VCM profile as a cell function $\delta \widetilde{\mathbf{m}}_{\mathbf{k}}(\mathbf{r})$ depending on the following existence conditions:

- For geometry E, in order for us to be able to generate the VCM, the field can be applied along either of the three independent directions, corresponding to the central axis of each of the three (indistinguishable) macrospins.
- For geometries B, C, and D (one out of three macrospins is disjointed): the field must be applied only along one particular direction corresponding to the central axis of the disjointed macrospin.

This becomes especially important in the context of magnonic crystals, where information is encoded in propagating spin waves, or magnons (e.g., Refs. [33-36]). An example of a magnonic crystal is an ASI structure consisting of periodic repetitions of the vertices we studied here. While it is outside the scopes of this paper, we conducted preliminary calculations of the bandwidth and average group velocity of a propagating $\mathrm{VCM}$ in a fully periodic ASI, made of Type E macrospin triads as unit cell using the primitive vectors $\mathbf{d}_{1}=(0,950 \mathrm{~nm})$ and $\mathbf{d}_{2}=(775 \mathrm{~nm}, 470 \mathrm{~nm})$ : as illustrated in Tab. I, the VCM Bloch wave has a group velocity of around $6.2 \mathrm{~m} / \mathrm{s}$ if the $\Gamma M$ direction is selected in the reciprocal space (corresponding to direction $\theta=0^{\circ}$ ). Note that the VCM bandwidth is limited according to the limited dynamic stray fields of the VCM, as a consequence of its peculiar localization. As we have shown, it is possible to deterministically switch the VCM mode on/off simply by tuning the magnetic field direction: as far as the full periodic ASI is concerned, this implies the switch of the corresponding SW propagation channel; even more, any variation in oscillation (periodic or random) of the sample-to-field angle would lead to a corresponding shift of the output FMR signal, which is itself an original way to encode an analog signal. In other words, one could design a sensor that correlates an intensity variation of the VCM to a misalignment of the angle between sample and field, and implement a feedback loop that realigns the sample until the original FMR intensity is recovered.

\section{ACKNOWLEDGEMENTS}

FM, supported in the calculations by RS, conceived the theoretical model and drove the simulation strategy for the interpretation of the data and the hypothesis (confirmed) of the VCM. Work in Ferrara included also manuscript preparation. Work at Delaware, including FMR measurements, data analysis, and manuscript preparation, was supported by the U.S. Department of Energy, Office of Basic Energy Sciences, Division of Materials Sciences and Engineering under Award DESC0020308. Work at Northwestern, including experimental design, was supported under NSF Grant No. DMR 1507058. Device fabrication including thin film growth was carried out at Argonne and supported by the U.S. Department of Energy (DOE), Office of Science, Materials Science and Engineering Division. Lithography was carried out at the Center for Nanoscale Materials, an Office of Science user facility, which is supported by DOE, Office of Science, Basic Energy Science under Contract No. DE-AC02-06CH11357. 
* Current address: Institute of Advanced Materials, LG Chem, Daejeon 34122, Korea

† Current address: Department of Materials Science and Engineering, University of Illinois at Urbana-Champaign, Urbana, Illinois 61801, USA

¥ montoncello@fe.infn.it

$\S$ mbj@udel.edu

1 H. Arava, N. Leo, D. Schildknecht, J. Cui, J. Vijayakumar, P. M. Derlet, A. Kleibert, and L. J. Heyderman, Physical Review Applied 11, 054086 (2019), 10.1103/PhysRevApplied.11.054086.

2 J. Drisko, T. Marsh, and J. Cumings, Nature Communications 8:14009, 1 (2017), 10.1038/ncomms14009.

3 E. Iacocca, S. Gliga, R. L. Stamps, and O. Heinonen, Physical Review B 93, 134420 (2016).

4 X. Zhou, G. L. Chua, N. Singh, and A. O. Adeyeye, Advanced Functional Materials 26, 1437 (2016).

${ }^{5}$ V. S. Bhat, F. Heimbach, I. Stasinopoulos, and D. Grundler, Physical Review B 93, 140401(R) (2016), 10.1103/PhysRevB.93.140401.

6 S. H. Skjærvø, C. H. Marrows, R. L. Stamps, and L. J. Heyderman, Nature Reviews Physics 439, 1 (2019).

7 J. Sklenar, S. Lendinez, and M. B. Jungfleisch, Dynamics in artificial spin ice and magnetic metamaterials, 1st ed., Solid State Physics, Vol. 70 (Elsevier Inc., 2019).

8 S. Lendinez and M. B. Jungfleisch, Journal of Physics: Condensed Matter 32, 013001 (2019).

9 W. Bang, F. Montoncello, M. T. Kaffash, A. Hoffmann, J. B. Ketterson, and M. B. Jungfleisch, Journal of Applied Physics 126, 203902 (2019).

10 V. Kapaklis and et al., New J. Phys. 14, 035009 (2012).

11 V. S. Bhat, J. Sklenar, B. Farmer, J. Woods, J. T. Hastings, S. J. Lee, J. B. Ketterson, and L. E. De Long, Physical Review Letters 111, 38 (2013)

12 S. A. Daunheimer, O. Petrova, O. Tchernyshyov, and J. Cumings, Physical Review Letters 107, 167201 (2011)

13 Y. Shen, O. Petrova, P. Mellado, S. Daunheimer, J. Cumings, and O. Tchernyshyov, New Journal of Physics 14, $035022(2012)$

14 B. Farmer, V. S. Bhat, A. Balk, E. Teipel, N. Smith, J. Unguris, D. J. Keavney, J. T. Hastings, and L. E. De Long, Physical Review B 93, 134428 (2016)

15 F. Montoncello, L. Giovannini, B. Farmer, and L. De Long, Journal of Magnetism and Magnetic Materials 423, 158 (2017)

16 J. Sklenar, Y. Lao, A. Albrecht, J. D. Watts, C. Nisoli, G.W. Chern, and P. Schiffer, Nature Physics 15, 191 (2019).

17 S. Gliga, G. Hrkac, C. Donnelly, J. Büchi, A. Kleibert, J. Cui, A. Farhan, E. Kirk, R. V. Chopdekar, Y. Masaki, N. S. Bingham, A. Scholl, R. L. Stamps, and L. J. Heyderman, Nature Materials 16, 1106 (2017).
18 S. Gliga, A. Kákay, R. Hertel, and O. G. Heinonen, Physical Review Letters 110, 117205 (2013).

19 T. Dion, D. M. Arroo, K. Yamanoi, T. Kimura, J. C. Gartside, L. F. Cohen, H. Kurebayashi, and W. R. Branford, Physical Review B 100, 054433 (2019), 10.1103/PhysRevB.100.054433.

20 E. Iacocca, S. Gliga, and O. Heinonen, arXiv.org , 1903.01887 (2019).

21 A. Vansteenkiste, J. Leliaert, M. Dvornik, M. Helsen, F. Garcia-Sanchez, and B. Van Waeyenberge, AIP Advances 4, 107133 (2014).

22 M. B. Jungfleisch, W. Zhang, E. Iacocca, J. Sklenar, J. Ding, W. Jiang, S. Zhang, J. E. Pearson, V. Novosad, J. B. Ketterson, O. Heinonen, and A. Hoffmann, Physical Review B 93, 100401 (2016).

${ }^{23}$ We refer to the mode that is the closest to the ideal uniform mode as fundamental mode; i.e., it displays the smallest number of nodal lines (lowest order mode), as well as oscillations involving a significant amount of the macrospin surface (bulk mode). Due to the non-ellipsoidal shape of the macrospins, the profile of this mode is sometimes calculated as hybridized, namely displaying undulations without actual phase change.

24 F. Montoncello, W. B. L. Giovannini, M. B. Jungfleisch, A. Hoffmann, J. B. Ketterson, B. W. Farmer, and L. E. D. Long, Physical Review B 97, 014421 (2018).

25 W. Bang, F. Montoncello, M. B. Jungfleisch, A. Hoffmann, L. Giovannini, and J. B. Ketterson, Physical Review B 99, 014415 (2019).

26 M. Grimsditch, G. K. Leaf, H. G. Kaper, D. A. Karpeev, and R. E. Camley, Phys. Rev. B 69, 174428 (2004).

27 C. Kittel, Physical Review 73, 155 (1948).

28 D. Kuźma, F. Montoncello, P. Sobieszczyk, A. Wal, L. Giovannini, and P. Zieliński, Journal of Applied Physics 124, 223902 (2018).

29 F. Montoncello and F. Nizzoli, Journal of Applied Physics 107, 023906 (2010)

30 C. Bayer, S. O. Demokritov, B. Hillebrands, and A. N. Slavin, Applied Physics Letters 82, 607 (2003)

31 G. Gubbiotti, L. L. Xiong, F. Montoncello, and A. O. Adeyeye, Appl. Phys. Lett. 111, 192403 (2017).

32 F. Montoncello and L. Giovannini, Appl. Phys. Lett. 104, 242407 (2014).

33 M. Krawczyk and D. Grundler, Journal of Physics: Condensed Matter 26, 123202 (2014).

34 A. V. Chumak, A. A. Serga, and B. Hillebrands, Journal of Physics D: Applied Physics 50, 244001 (2017).

35 V. V. Kruglyak, S. O. Demokritov, and D. Grundler, Journal of Physics D: Applied Physics 43, 264001 (2010).

36 B. Lenk, H. Ulrichs, F. Garbs, and M.Münzenberg, Physics Reports 507, 107 (2011). 